\title{
Wenn Medien und Gesellschaft verschmelzen: Warum Marx als Medientheoretiker entdeckt wird.
}

Es ist sicherlich wenig überraschend, dass Karl Marx und marxistische Theorien sich in der derzeitigen tiefgreifenden Krise des Kapitalismus großer Beliebtheit erfreuen. Dass seine Beliebtheit in den letzten beiden Jahrzehnten auch auf die Medienund Kommunikationstheorie übergegriffen ist, scheint hingegen erklärungsbedürftig. Denn dies war beileibe nicht immer so. So hat etwa in den 1970er Jahren der kanadische Medienforscher Dallas Smythe die Analyse von Medien und Kommunikation als einen ‘blind spot<, als toten Winkel der westlichen marxistischen Theorie verstanden. ${ }^{\mathrm{I}}$

Ist Marx auch ein Medien- und Kommunikationstheoretiker? Smythe hat diese Frage nicht explizit gestellt. Er hätte wohl geantwortet, dass Marx in erster Linie ein Sozial- und Wirtschaftstheoretiker ist, dass jedoch seine politische Ökonomie und seine materialistische Methode eine kaum zu überschätzende Ressource zur Analyse von Medien und Kommunikation darstellen.

Obwohl ich einer solchen Sicht im Großen und Ganzen zustimme, ist die Frage von Marx als einem Medientheoretiker im digitalen Kapitalismus damit noch nicht hinreichend beantwortet. Denn zwischen den 1970er Jahren und heute haben sich Medien und Kommunikation so gravierend verändert, dass sich meines Erachtens die Frage erübrigt, ob Marx ein Medientheoretiker ist. Oder anders formuliert: Im digitalen Kapitalismus sind Information und Kommunikation so sehr ins Zentrum aller sozialen und wirtschaftlichen Prozesse gerückt, dass eine klare konzeptionelle Trennung zwischen den Feldern Medien und Gesellschaft nicht mehr produktiv ist. Im digitalen Kapitalismus ist Gesellschaft schlichtweg nicht mehr ohne Informations- und Kommunikationstechnologien zu denken. Und umgekehrt sind Medien heute nicht mehr nur Artefakte mit Inhalten und Bedeutungen, sondern soziale und ökonomische Regime.

Dieser Beitrag hat drei Teile. Im ersten Teil wird die Relevanz von Marx für die Analyse von Massenmedien erörtert. Es wird gezeigt, dass und warum im Zeitalter der Massenmedien die Reichweite marxistischer Konzepte in der kritischen Medientheorie eher begrenzt war. Im zweiten Teil wird die These entwickelt, warum im di-

I Dallas Smythe, »Communications. Blindspot of Western Marxism«, in: Canadian Journal of Political and Social Thought, I. Jg., 3/1977, S. I-27. 
gitalen Kapitalismus Medien und Gesellschaft verschmelzen. Im dritten Teil werden dann die Auswirkungen dieser Entwicklung für eine marxistische Analyse des digitalen Kapitalismus diskutiert. Dieser Teil ist der eigentliche Kern des Beitrags. Hier soll gezeigt werden, warum jetzt alle zentralen Konzepte marxistischer Theoriebildung zur Analyse von digitalen Medien und Technologien fruchtbar gemacht werden können.

DIE POLITISCHE ÖKONOMIE VON MEDIEN UND KOMMUNIKATION IM ZEITALTER DER MASSENMEDIEN

Die politische Ökonomie von Medien und Kommunikation hat sich zu einer Zeit als akademisches Forschungsfeld konstituiert, als Medien in erster Linie Massenmedien waren. Massenmedien - also Fernsehen, Radio und Zeitungen - sind (oder waren bis in die 1990er Jahre) lineare Medien, die auf einem einseitigen Kommunikationsfluss basieren. Diese einseitige Kommunikation verläuft von zumeist wenigen Produzenten medialer Inhalte zu vielen Rezipienten, also zur Masse. ${ }^{2}$ Im Zeitalter der Massenmedien war klar, was Medien sind, und was Medientechnologien sind. Im Zeitalter der Massenmedien hat also die konzeptionelle Trennung von Medien und Gesellschaft noch funktioniert.

Einem breiten Konsens zufolge - was dieses Feld im Kern verbindet und was die zentralen Themen, Probleme und Fragestellungen sind - beginnt das Grundproblem mit der Beobachtung, dass Medienorganisationen im letzten Drittel des vorigen Jahrhunderts zunehmend privatisiert wurden, dass sie zu Medienunternehmen transformiert wurden. ${ }^{3}$ Besonders deutlich war dieser Prozess in den 1980er Jahren zu beobachten, als Satellitentechnologien eine Vielzahl neuer privater Fernsehkanäle hervorbrachten. Diese Entwicklung wurde als problematisch angesehen, weil Medienindustrien nicht wie andere Industrien sind. Medienindustrien weisen - verglichen mit anderen Industrien - eine Besonderheit auf: Die Produkte der Medienindustrien sind gleichzeitig Waren und öffentliche Güter. Sie sind Waren, weil die Konsumenten für diese Produkte bezahlen und weil die Besitzer dieser Industrien damit Profite erzielen. Sie sind öffentliche Güter, weil diese Produkte maßgeblich die öffentliche Sphäre (im Zeitalter der Massenmedien) konstituieren. Dies gilt natürlich insbesondere für Nachrichten. Medienindustrien haben also zum einen eine soziale, kulturelle, und politische Funktion, zum anderen haben sie ökonomische Interessen und streben nach Gewinnmaximierung. Eben dieser janusköpfige Charakter von Medienindustrien verdeutlicht, wie fragwürdig es ist, Medien als unabhängige Organisationen

2 Mark Poster, The Second Media Age, Cambridge: Polity Press 1995.

3 Vincent Mosco, The Political Economy of Communication. Rethinking and Renewal, London: Sage 1996. 
zu verstehen, die im öffentlichen Interesse operieren und eine demokratiestützende Funktion haben.

Die politische Ökonomie von Medien und Kommunikation geht also davon aus, dass Medien machtvoll sind, weil sie einen sehr starken Einfluss auf die öffentliche Meinungsbildung und den öffentlichen Diskurs haben. Deshalb ist es wichtig, den politischen und ökonomischen Kontext der Medieninhalte zu analysieren, also deren Produktionsbedingungen zu studieren.

Die folgenden Themen stehen im Zentrum des Forschungsinteresses. Zunächst geht es um ein Verständnis von Medienindustrien als Markt. Wie erzeugen Medienunternehmen Einkommen, wie generieren sie Profit? Des Weiteren geht es um eine Analyse der Eigentumsbedingungen von Medienorganisationen (von öffentlichen, privaten und gemeinnützigen Medienorganisationen). Ebenfalls zentral sind Fragen nach Transformationen der Medienorganisationen, also etwa Analysen zur Internationalisierung von Medienorganisationen, Analysen zur Diversifizierung von Medienprodukten, oder Analysen zur Konzentration von Medienorganisationen und die damit einhergehende Entstehung von global operierenden Medienkonzernen. Schließlich befasst sich das Feld mit der nationalen und internationalen Regulierung von Massenmedien.

Die Transformationen der Massenmedien, so der generelle Konsens im Feld, sind in vielerlei Hinsicht besorgniserregend. Immer mehr Medieninhalte werden zu Waren, alternative Medieninhalte werden tendenziell immer mehr unterdrückt, die Logik des Kapitals dominiert, und das öffentliche Interesse wird dem privaten Interesse untergeordnet.

Der marxistische Kulturtheoretiker Raymond Williams war sicherlich einer der ersten, der diese Entwicklungen bereits Anfang der 1960er Jahre erkannt hat. Er beginnt einen Artikel zum Wachstum der Zeitungsindustrie in England mit der Beobachtung, dass kultur- und sozialgeschichtliche Analysen es weitgehend versäumt haben, die Geschichte der Presse mit einer Wirtschaftsgeschichte zu verbinden, um dann eine solche Analyse zu erstellen, eine empirische Untersuchung, die einen Zeitraum von 170 Jahren erfasst. Seine Ergebnisse sind ernüchternd und nehmen einen Großteil der Debatten vorweg, die Jahrzehnte später mit großem Engagement geführt werden. ${ }^{4}$

Inwiefern basiert diese Perspektive der politischen Ökonomie von Massenmedien auf einer marxistischen Analyse? Viele der Texte, die im Feld als Klassiker gelten, haben entweder gar keine Verweise auf Marx oder nur sehr wenige. ${ }^{5}$ In letzterem Fall dienen die Verweise eher als grobe Markierungspunkte, um etwa den liberalen Strang der politischen Ökonomie von der marxistischen zu unterscheiden.

4 Raymond Williams, The Long Revolution, London: Chatto \& Windus r96r.

5 Andreas Wittel, Digital Marx. Toward a Political Economy of Distributed Media«, in: tripleC, , Jg., 2/2012, S. 3I3ff. 
Dennoch sind all diese Texte von Marx beeinflusst oder, um genauer zu sein, vom Basis-Überbau-Konzept und von einer materialistischen Analyse von Medien und Kommunikation. Bekanntlich basiert seine Gesellschaftsanalyse auf einer Unterscheidung von Basis und Überbau. Die Basis bezieht sich auf die materiellen Produktionsbedingungen. Zur Basis gehören die Produktivkräfte (also etwa Technologien) und die Klassenbeziehungen (im Kapitalismus sind dies Kapitalisten und Lohnarbeiter) in einer Gesellschaft. Der Überbau bezieht sich auf nicht-materielle Aspekte einer Gesellschaft, also etwa deren Kultur und deren Religion, deren Ideen, Werte und Normen. Zwar ist die Beziehung von Basis und Überbau grundsätzlich gegenseitig und dialektisch (wie alles bei Marx), letztendlich ist es jedoch die materielle Basis, die den ideellen Überbau determiniert (die große Ausnahme dieser Regel sind natürlich Revolutionen oder soziale Bewegungen).

Die Gedanken der herrschenden Klasse sind in jeder Epoche herrschende Gedanken, d. h. die Klasse, welche die herrschende materielle Macht der Gesellschaft ist, ist zugleich ihre herrschende geistige Macht. Die Klasse, die die Mittel zur materiellen Produktion zu ihrer Verfügung hat, disponiert damit zugleich über die Mittel zur geistigen Produktion, so daß ihr damit zugleich im Durchschnitt die Gedanken derer, denen die Mittel zur geistigen Produktion abgehen, unterworfen sind. Die herrschenden Gedanken sind weiter Nichts als der ideelle Ausdruck der herrschenden materiellen Verhältnisse. ${ }^{6}$

Zwar setzen sich einige der Klassiker (etwa Nicholas Garnham und Graham Murdock) auch direkt und ausführlich mit Marx auseinander, allerdings wird dann zumeist gegen eine deterministische Auslegung des Basis-Überbau Konzepts argumentiert.7

Der Punkt, auf den es mir hier vor allem ankommt: die politische Ökonomie von Massenmedien hat nur sehr begrenzt auf das theoretische Besteck der politischen Ökonomie von Karl Marx zurückgegriffen. Dies ist jedoch keineswegs als Kritik zu verstehen. Ich denke vielmehr, dass diese Einschränkung im Zeitalter der Massenmedien der Logik der Technologien von Massenmedien entsprach. Um zu verstehen, was sich in den letzten Jahrzehnten verändert hat, ist es unerlässlich, die Entwicklung von Massenmedien zu digitalen und distributiven Medien zu verstehen.

6 Karl Marx/Friedrich Engels, Die deutsche Ideologie, MEW 3, Berlin: Dietz 1985, S. 46.

7 Nicholas Garnham, Capitalism and Communication: Global Culture and the Economics of Information, London: Sage 1990. Graham Murdock, »Large Corporations and the Control of Communications Industries«, in: Culture, Society and the Media, hrsg. v. Michael Gurevitch, London: Methuen I982, S. II8-I50. 
Im Zeitalter der Massenmedien ließen sich die Medienindustrien und die Kulturindustrien klar von anderen Industrien (etwa der Automobilindustrie) unterscheiden. Medien- und Kulturindustrien haben Nachrichten, Unterhaltung und kulturelle Produkte (Bücher, Filme, Lieder, Fernsehserien etc.) produziert, während die Automobilindustrie Autos hergestellt und die Tourismusindustrie Urlaube angeboten hatte. Diese klare Abgrenzung zwischen unterschiedlichen industriellen Sektoren basierte zwar auch auf einer klaren Unterscheidung zwischen unterschiedlichen Produkten, die hergestellt wurden (Medien und Kultur versus Autos), vor allem aber auf sehr verschiedenen Technologien, die zur Herstellung eben dieser Produkte notwendig waren.

Eben dies hat sich mit der Digitalisierung aller Technologien und aller industriellen Sektoren grundlegend verändert. Erst im Zeitalter digitaler Technologien ist es möglich, dass etwa der Apple-Konzern, der in den 1980er und 1990er Jahren (also in der Hochphase der Massenmedien) ein klassischer Computerhersteller war und mit Musik nichts zu tun hatte, wenig später der herausragende Marktführer in der $\mathrm{Mu}^{-}$ sikbranche werden konnte. Die Firma Kodak war kein Medienunternehmen, sondern im Fotogeschäft aktiv. Die Firma Instagram, der vielleicht logische Nachfolger von Kodak, wird dagegen eindeutig im Bereich der Medien- und Kreativindustrien verortet. Google hat 1998 als Softwareunternehmen begonnen, wurde jedoch wenige Jahre später als eines der weltweit größten Medienunternehmen gehandelt. Damit nicht genug, hat der Konzern seine Produktpalette weiter ausgebaut und operiert jetzt in Bereichen, die mit traditionellen Medienindustrien nur wenige Überschneidungspunkte haben. Dies zeigt sich etwa am Beispiel von Googles beträchtlichen Investitionen in Bereichen wie Robotics und künstlicher Intelligenz. Dies zeigt sich auch am Beispiel der Herstellung des fahrerlosen Autos. Ist Google damit zu einem Automobilhersteller geworden? Kann Google in Zukunft den Automobilmarkt dominieren, ähnlich wie Apple die Musikindustrie? Ist das fahrerlose Auto zuallererst ein Auto, oder ist es vor allem ein Computer in Gestalt einer Autokarosserie und vier Reifen? Ist Bitcoin, die erste rein digitale Geldform, ein Produkt der Finanzindustrie oder der Medienindustrie? Ist Uber ein Transportunternehmen oder ein Mediengigant? Gehört Airbnb zur Medienindustrie oder in die Tourismusbranche?

Diese Fragen und Beispiele zeigen, wie brüchig die ursprüngliche Trennlinie zwischen Medienindustrien und anderen Industrien inzwischen geworden ist. Für fast jeden Bereich wirtschaftlicher Aktivität gibt es inzwischen eine Plattform, die hierfür eine App entwickelt hat, die Dienstleistungen anbietet und zumeist auf globaler Ebene operiert. Genau hierin unterscheidet sich der kommunikative Kapitalismus vom Kapitalismus im Zeitalter der Massenmedien. Die Druckerpresse war ausschließlich eine Medientechnologie. Dies gilt auch für die Nutzung der Radiowellen oder für die 
Fernsehröhre. Digitale Technologien sind jedoch nicht nur Medientechnologien. Sie sind eingebettet in fast alle Produkte und integraler Bestandteil fast aller Industrien. Manuel Castells war sicherlich einer der ersten, der diese neue Rolle der Informations- und Kommunikationstechnologien erkannt und analysiert hat.

What characterises the current technological revolution is not the centrality of knowledge of information, but the application of such knowledge and information to knowledge generation and information processing/communication devices in a cumulative feedback loop between innovation and the uses of innovation. ${ }^{8}$

Eben diese Relation zwischen Wissensproduktion und Information und die hieraus entstehenden Rückkopplungsschleifen haben zu einer immer größeren Beschleunigung technologischen Wandels geführt. Castells nennt fünf Merkmale des informationstechnologischen Paradigmas. Die beiden ersten Merkmale sind für mein Argument besonders relevant:

The first characteristic of the new paradigm is that information is its raw material: these are technologies to act on information, not just information to act on technology, as was the case in previous technological revolutions. The second feature refers to the pervasiveness of effects of new technologies. Because information is an integral part of all human activity, all processes of our individual and collective existence are directly shaped (although certainly not determined) by the new technological medium. ${ }^{9}$

Im digitalen Zeitalter ist Information selbst zu einem Produkt geworden. Daraus leitet er den grundsätzlichen Unterschied ab zwischen der Industriegesellschaft und der Informationsgesellschaft. In der Informationsgesellschaft operieren alle industriellen Sektoren (unter anderem Landwirtschaft, Manufaktur, Dienstleistung, Finanzwirtschaft) auf der Basis von digitalen Technologien.

Dass digitale Technologien nicht nur Medientechnologien sind, sondern Technologien, die in allen Feldern wirtschaftlicher Aktivität eine ganz zentrale Rolle einnehmen, ist allerdings nur eine Erklärung für die Verschmelzung von Medien und Gesellschaft. Der zweite, ebenso wichtige Grund hängt mit der Allgegenwart und der Omnipräsenz digitaler Medien zusammen. Wenn alle, die mit einem Computer oder einem Smartphone ausgestattet sind, potenziell Informationen produzieren und weiterleiten können, sind Medien eben nicht mehr eine externe Institution, sondern ein integraler Teil unseres Lebens. Wenn die Produktion und Konsumption von Medieninhalten in

8 Manuel Castells, The Rise of the Network Society, Maldan, MA: Blackwell I996, S. 3I.

9 Ebd., S. 70. 
'produsager münden, wenn es darüber hinaus keine Zeiten und Orte für sprodusages gibt, dann sind Medien so integriert in unser Leben, unser Zusammenleben und unsere Zusammenarbeit, dass es immer weniger Sinn macht, Medien als einen spezifischen Bereich von Gesellschaft zu verstehen. Digitalisierung ist der Moment, in dem Medien sich so in alle Aspekte unseres Lebens und unserer Gesellschaft eingenistet haben, dass sie letztendlich von diesen analytisch nicht mehr zu trennen sind.

Was bedeutet diese Entwicklung für die politische Ökonomie von Medien und Kommunikation? Im digitalen oder kommunikativen oder kognitiven Kapitalismus mündet die politische Ökonomie von Medien und Kommunikation zwangsläufig in eine allgemeine politische Ökonomie, die sich, um ein Beispiel zu nennen, ebenso für die Finanzindustrie wie für die Medienindustrie interessiert, die dann etwa Bitcoin als ein Phänomen versteht, das vieles ist, Netzwerk, Plattform, Medium, Technologie, Information, Datenbank und Geld.

Was bedeutet diese Entwicklung für Medientheorie und Marx? Ich habe bereits gezeigt, dass die politische Ökonomie von Medien und Kommunikation im Zeitalter der Massenmedien nur sehr begrenzt auf das theoretische Besteck von Marx zurückgreifen konnte. In dieser Epoche reduzierte sich der Nutzen von Marx weitgehend auf das Basis-Überbau-Modell, mit dessen Hilfe kritische Medientheorie erklären konnte, warum die Frage des Eigentums von (Medien-)Produktionsmitteln so entscheidend ist für die Analyse des ideologischen Charakters von Medieninhalten. Im Zeitalter digitaler Medientechnologie oder im Zeitalter des kommunikativen Kapitalismus bleiben das Basis-Überbau-Modell und die Frage nach dem Eigentum an Produktionsmitteln nach wie vor relevant. Allerdings ist dies nicht mehr die einzige Brücke zur marxistischen politischen Ökonomie. Jetzt wird die Gesamtheit marxistischer Theoriebildung wichtig, jetzt kann der gesamte Kanon marxistischer Konzepte produktiv gemacht werden. Dies werde ich im letzten Teil meines Beitrags an fünf zentralen marxistischen Begriffen veranschaulichen, an den Begriffen Ware, Arbeit, Wert, Eigentum, und Klassenkampf.

\section{WARE}

Bekanntlich ist die Ware, wie Marx im ersten Satz von Das Kapital 1 erklärt, die Elementarform der kapitalistischen Produktionsweise. Im digitalen Kapitalismus gibt es zwei Entwicklungen, die eine genauere Beschäftigung mit dem Konzept der Ware nahelegen.

Die erste Entwicklung bezieht sich auf die Herausbildung der sogenannten sdigital commonss, also jener Bereiche des Internets, die als Gemeingüter produziert und erhalten werden, die also explizit nicht für den Markt produziert werden. Hierzu 
zählen etwa Software-commons (also free and open-source code), Wissens-commons (das bekannteste Beispiel hierfür ist Wikipedia, hierzu gehören jedoch auch akademische open-access Zeitschriften), Nachrichten-commons (bekannte Beispiele hierfür sind etwa die Hinweisgeberplattform Wikileaks und die einstündige Internetsendung DemocracyNow, sowie zahlreiche Online-Magazine im Bereich alternativer Medien), Kommunikations- und Interaktions-commons (etwa Mailing-Listen wie nettime oder das soziale Netzwerk Diaspora), Kunst-commons (etwa Rhizome, ubu.web oder netartnet.net) sowie all die Texte, Bilder, Filme und Musikstücke, die unter CreativeCommons-Lizenzen verbreitet werden. Digital commons sind also ein Bereich im Internet, der weder unter staatlicher Kontrolle steht, noch Teil der Warenwirtschaft ist. Damit stehen >digital commons für eine Entwicklung, die ich vor einigen Jahren als >counter-commodification ‘ charakterisiert habe. ${ }^{\text {Io }}$ Während Kommodifizierung einen Prozess bezeichnet, in dem Dinge oder Dienstleistungen, die zuvor nicht Teil der Warenwirtschaft waren, zu Waren werden, steht Gegen-Kommodifizierung für den umgekehrten Prozess, also für eine Entwicklung, in der Dinge oder Dienstleistungen, die bereits in Warenform existieren, in Gemeingüter umgewandelt werden. Die oben genannten Bereiche des >digital commons` sind allesamt Beispiele für Gegen-Kom-

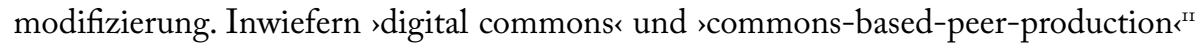
jedoch der Keim für eine neue postkapitalistische Ökonomie sein können, bleibt derzeit eher fraglich. Denn >digital commons sind eine kleine Insel mitten im kapitalistischen Meer, das sich aufgrund einer zweiten Entwicklung sogar noch machtvoll auszudehnen scheint.

Diese zweite Entwicklung ist eine Kommodifizierung von Informationen in Form von Daten. Dies sind vor allem Daten über Internetnutzer, die mittels digitaler Technologien auf diversen Plattformen wie Google, Facebook oder Twitter kommunizieren und interagieren. Diese Daten haben einen enorm hohen Tauschwert - sie werden vor allem an Anzeigenkunden verkauft. Wir, die Nutzer dieser Plattformen, sind keine Kunden, sondern liefern mit unseren Daten, die wir den neuen Unternehmen des Informationszeitalters kostenlos zur Verfügung stellen, die Rohmaterialien, die gesammelt, geordnet, analysiert und schließlich verkauft werden. Man könnte fragen, ob es sich hierbei um eine Form der ursprünglichen Akkumulation handelt, also um eine gewaltsame Enteignung unserer Produktionsmittel. Warum gehören unsere Daten Facebook und nicht uns? Seit kurzem werden erste Forderungen laut, die sogenannten >data centers unter staatliche Kontrolle zu bringen. ${ }^{\text {I2 }}$

Io Andreas Wittel, »Counter-Commodification. The Economy of Contribution in the Digital Commons«, in: Culture and Organization, 19. Jg., 4/2013, S. 314-331.

II Yochai Benkler, The Wealth of Networks. How Social Production Transforms Markets and Freedom, New Haven: Yale University Press 2006.

I2 Evgeny Morozov, »Socialize the Data Centers«, in: New Left Review, 91/2015, S. 45-66. 


\section{ARBEIT}

Für Marx ist Arbeit bekanntlich einer der zentralen Grundbegriffe seiner Philosophie. Arbeit ist für ihn nicht nur eine wirtschaftliche, sondern eine menschliche Tätigkeit. Sie ist eine Universalkategorie der menschlichen Existenz und ist als solche unabhängig von spezifischen sozialen und ökonomischen Formen. »Die Arbeit ist zunächst ein Prozess zwischen Mensch und Natur, ein Prozess, worin der Mensch seinen Stoffwechsel mit der Natur durch seine eigene Tat vermittelt, regelt und kontrolliert. « $^{13}$

Im Unterschied zur Arbeit verweist der Arbeitsprozess auf wirtschaftliche und soziale Besonderheiten, auf je spezifische Produktionsformen. Transformationen von Arbeitsprozessen sind dann immer auch Transformationen von Gesellschaften und Ökonomien. Der kapitalistische Arbeitsprozess basiert in erster Linie auf Lohnarbeit.

Postoperaistische Theoretiker haben Ende der 1990er Jahre mit der These Aufsehen erregt, dass Lohnarbeit nicht mehr das alleinige Kriterium für Wertschöpfung und Profitbildung ist. Seitdem immaterielle Arbeit in post-fordistischen Gesellschaften ins Zentrum der Produktion gerückt ist, so das Argument, werde gesellschaftlicher Wert auch jenseits von Lohnarbeit hergestellt. Im kognitiven Kapitalismus sei Arbeit nicht mehr unter den Bedingungen traditioneller Fabrikarbeit zu verstehen.

It is not simply that intellectual labor has become subjected to the norms of capitalist production. What has happened is that a new 'mass intellectuality has come into being, created out of a combination of the demands of capitalist production and the forms of sself-valorization that the struggle against work has produced. ${ }^{14}$

Diese Befunde haben, angestoßen von Tiziana Terranova, zu einer Debatte um sfree labour, also um unbezahlte Arbeit geführt. "Free labour is the moment where this knowledgeable consumption of culture is translated into excess productive activities that are pleasurably embraced and at the same time often shamefully exploited. $\varkappa^{15} \mathrm{Wie}$ allerdings diese Ausbeutung funktionieren soll, wird weder bei Terranova erklärt, noch bei anderen, die diese Debatte aufgegriffen und weitergeführt haben.

In jüngster Zeit haben sich marxistische Medientheoretiker auch den materiellen Formen von digitaler Arbeit zugewandt. Sowohl Christian Fuchs als auch Nick Dyer-Witheford liefern eine hervorragende Analyse zu allen Formen digitaler Arbeit, von der Minenarbeit im Kongo etwa zur Extraktion von Coltan, zur Manufaktur

I3 Karl Marx, Das Kapital. Band I, MEW 23, Berlin: Dietz 1985, S. 192.

I4 Maurizio Lazzarato, »Immaterial Labour«, in: Generation Online, http://www.generation-online. $\mathrm{org} / \mathrm{c} /$ fcimmateriallabour3.htm, 1996 (03. 02. 2018).

I5 Tiziana Terranova, Network Culture. Politics for the Information Age, London: Pluto Press 2004, S. 78 . 
von Halbleitern im Silicon Valley, dem Outsourcing zur Herstellung der Hardware in Südostasien, der Produktion von Software, der Arbeit in Callcentern in Indien. ${ }^{16}$ Diese Analysen machen zum einen deutlich, wie weitreichend das Konzept der digitalen Arbeit inzwischen ist. Zum anderen verweisen sie - in bester marxistischer Tradition - auf die Relevanz der materiellen Dimensionen von digitaler Arbeit.

\section{WERT}

Wie entsteht Wert, oder genauer, Tauschwert? Dies ist sicherlich eine der brennendsten Fragen, um Wertschöpfung im digitalen Kapitalismus zu verstehen. Marx bietet zwei Antworten.

In Kapital 1 entwickelt er die sogenannte Arbeitswerttheorie. Demnach berechnet sich der Wert eines Produkts oder einer Dienstleistung aus der menschlichen Arbeit, die zur Herstellung benötigt wird. Oder genauer, der Wert bestimmt sich über die durchschnittliche gesellschaftliche Arbeitszeit zur Herstellung dieser Ware. Damit setzt sich Marx ausdrücklich gegen liberale Werttheorien ab, denen zufolge der Tauschwert im Prozess des Tauschs festgelegt wird. Selbst in marxistischen Debatten wird die Arbeitswerttheorie sehr kontrovers diskutiert. So meint etwa Slavoj Žižek, die Arbeitswerttheorie sei »usually considered the weakest link in the chain of Marx's theory «. ${ }^{17}$

In der Tat lässt sich mit der Arbeitswerttheorie die Wertschöpfung im digitalen Kapitalismus nur sehr unzureichend erklären. Schließlich werden die Daten, mit denen etwa Facebook einen solch spektakulären Marktwert erzielt hat, nicht von Facebooks Angestellten produziert, sondern von den Nutzern der Plattform. Eine Werttheorie, die sich also nur auf Lohnarbeit konzentriert, kann meines Erachtens solche Phänomene nicht mehr schlüssig erklären.

What has irreversibly changed, however, from the times of the predominance of the classical theory of value, involves the possibility of developing the theory of value in terms of economic order, or rather, the possibility of considering value as a measure of concrete labor. ${ }^{18}$

Deshalb hat in den letzten beiden Jahrzehnten eine zweite Werttheorie von Marx an Popularität gewonnen. Insbesondere Theoretiker der Postoperaismo-Schule haben sich auf diese Werttheorie bezogen. In den Grundrissen, vor allem im >Fragment über

I6 Nick Dyer-Witheford, Cyber-Proletariat. Global Labor in the Digital Vortex, London: Pluto 2015.

Christian Fuchs, Digital Labour and Karl Marx, New York: Routledge 2015.

I7 Slavoj Žižek, Living in the End Times, London: Verso 20II, S. 205.

I8 Antonio Negri, »Value and Affect«, in: boundary 2, 26. Jg., 2/1999, S. 77-88, hier S. $77 f$. 
Maschinen, ist Wertschöpfung nicht nur ein Produkt von Lohnarbeit, sondern auch ein Produkt technologischer und wissenschaftlicher Entwicklung.

Die Aneignung der lebendigen Arbeit durch das Kapital erhält in der Maschinerie auch nach dieser Seite hin eine unmittelbare Realität: Es ist einerseits direkt aus der Wissenschaft entspringende Analyse und Anwendung mechanischer und chemischer Gesetze, welche die Maschine befähigt, dieselbe Arbeit zu verrichten, die früher der Arbeiter verrichtete. Die Entwicklung der Maschinerie auf diesem Weg tritt jedoch erst ein, sobald die große Industrie schon eine höhere Stufe erreicht hat und die sämtlichen Wissenschaften in den Dienst des Kapitals gefangengenommen sind. ${ }^{19}$

Diese zweite Werttheorie, in der der sgeneral intellect ‘ als wichtige Komponente der Wertbildung betont wird, ist sicherlich weit besser geeignet für eine Analyse des digitalen Kapitalismus, für einen Kapitalismus, der eine shöhere Stufe « erreicht hat. Dennoch ist auch diese Erklärung nicht unproblematisch. Zu kritisieren ist hier vor allem, dass diese Erklärung zu vage und allgemein bleibt und wenig Hilfe anbietet, wie dieses Zusammenspiel von Technologie, Wissen, und Subjektivität genauer zu verstehen ist. Es ist meines Erachtens eine der wichtigsten Aufgaben zeitgenössischer marxistischer Medientheorie, dieses Zusammenspiel besser zu veranschaulichen.

\section{EIGENTUM}

Von Marx können wir erstens lernen, dass Eigentum kein natürliches Phänomen ist, sondern ein soziales Konstrukt. Zweitens ist die Perspektive interessant, mit der er Eigentum theoretisiert. Marx analysiert nämlich nicht die Beziehung zwischen dem Objekt des Besitzes und der Besitzerin. Stattdessen theoretisiert er Eigentum als ein Verhältnis, über das der Besitzer von Eigentum seine Beziehung zu anderen Menschen definiert. Für Marx sind deshalb Eigentumsverhältnisse nichts anderes als ein Ausdruck von Klassenbeziehungen. In kapitalistischen Gesellschaften basiert Eigentum auf dem Gegensatz von Lohnarbeit und Kapital sowie auf der Anhäufung von Profit der Eigentümer von Produktionsmitteln. Im Kapitalismus ist Privateigentum also in erster Linie eine Form von Eigentum, das andere ausschließt. Schließlich sind Eigentumsbeziehungen ein Produkt spezifisch historischer Bedingungen.

Die französische Revolution z. B. schaffte das Feudaleigentum zugunsten des bürgerlichen ab. Was den Kommunismus auszeichnet, ist nicht die Abschaffung des Eigentums überhaupt, sondern die Abschaffung des bürgerlichen Eigentums [...]. In diesem Sinn

I9 Karl Marx, Grundrisse der Kritik der politischen Ökonomie, MEW 42, Berlin: Dietz 1985, S. 599. 
können die Kommunisten ihre Theorie in dem einen Ausdruck: Aufhebung des Privateigentums, zusammenfassen. ${ }^{20}$

Marx unterscheidet bekanntlich zwischen persönlichem und privatem Eigentum. Privates Eigentum im Kapitalismus lehnt er ab, denn dies ist lediglich ein Produkt entfremdeter und ausbeutender Arbeit. Privates Eigentum ist produktives Eigentum (Eigentum von Produktionsmitteln), es dient zur Erzeugung von Mehrwert. Demgegenüber ist persönliches Eigentum unproduktiv. Es dient dem Lebensunterhalt und der Reproduktion. Daher gibt es laut Marx keinen Grund, persönliches Eigentum zu sozialisieren.

Im Zeitalter der Massenmedien waren Copyright und geistiges Eigentum keine Themen, die in der Medienforschung eine wichtige Rolle gespielt haben. Dies hat sich mit dem Aufkommen des ssocial web grundlegend verändert. Zum einen sind die Technologien, die zur Produktion von Medieninhalten notwendig sind, deutlich billiger geworden. Keine Druckerpresse, kein Verlag, kein Fernsehsender, kein Aufnahmestudio - ein Laptop oder ein Mobiltelefon reichen völlig aus. Zum anderen können digitale Produkte (ein Buch, ein Song, ein Film etc.) zu geringen Kosten endlos reproduziert werden. Diese Möglichkeit der unbegrenzten Multiplikation macht digitale Produkte zu ınon-rival goods`, also zu Waren, die nicht durch Knappheit gekennzeichnet sind, und diese unterscheiden sich so grundlegend von einem auf Papier gedruckten Buch oder einer CD, die auf Knappheit beruhen und die etwa, wenn sie von den Eigentümern weggegeben werden, für diese nicht mehr verfügbar sind.

Die Diskussion zu Copyright und geistigem Eigentum wurde maßgeblich von Lawrence Lessig und Yochai Benkler angestoßen. ${ }^{21}$ Beide Rechtswissenschaftler argumentieren nicht grundsätzlich gegen Copyright, sondern wollen die gesetzlichen Beschränkungen von geistigem Eigentum deutlich reduzieren. Außerdem begrüßen sie die Entwicklungen im ssocial web<, die die Produktion und Zirkulation von freien (im Sinne von unbezahlten) kulturellen Werken ermöglicht haben. Im Kapitalismus selbst sehen sie kein Problem. Sie sehen auch nicht, dass die unendliche Reproduktion von kulturellen Gütern per digitalen Technologien für Kulturschaffende ein Problem erzeugt. Insofern gehören beide nicht zum marxistischen, sondern zum liberalen Flügel der politischen Ökonomie.

Mit Marx lässt sich diese Debatte in mehrfacher Hinsicht bereichern. Zum einen wäre darauf hinzuweisen, dass vieles von dem, was heute per intellektuellem Eigentum geschützt wird, kein persönliches Eigentum darstellt, sondern privates Eigentum. Im

20 Karl Marx/Friedrich Engels, Ausgewählte Werke, Moskau: Progress I982, S. 46.

2I Lawrence Lessig, Code and Other Laws of Cyberspace, New York: Basic Books 1999. Lawrence Lessig, Free Culture. The Nature and Future of Creativity, New York: Penguin 2004. Benkler, The Wealth of Networks. 
Informationszeitalter sind Information, Kommunikation und Wissen eben nicht nur Produkte, sondern immer auch Produktionsmittel. Dies gilt nicht nur für künstlerische oder wissenschaftliche Werke, sondern auch für `big data‘, für die Fülle an Daten, die entstehen, indem wir digitale Technologien benutzen.

Vielleicht noch wichtiger: Bis auf wenige Ausnahmen haben sich diese Debatten auf geistiges Eigentum beschränkt. Es ist dringend notwendig, diese Diskussion auf materielle Formen von Eigentum auszuweiten. Im digitalen Kapitalismus ist es weder konzeptionell noch politisch sinnvoll, geistiges Eigentum von materiellem Eigentum zu trennen. Oder mit Alain Badiou: Why do we »keep tight controls on all forms of property in order to ensure the survival of the powerful? « ${ }^{22}$

\section{KLASSENKAMPF}

Dyer-Witheford formuliert recht lustig, dass es ihm nicht nur um Kritik geht, sondern um Rebellion. Er erklärt zunächst, warum der Begriff `Klassenkampf ein solches Tabu geworden ist, dass er zumeist nur noch benutzt wird, wenn es darum geht, auf die Nutzlosigkeit dieses Begriffs hinzuweisen. Diejenigen jedoch, die mit dem Klassenbegriff in kritischer Hinsicht als Analysekategorie arbeiten, würden bestenfalls als reduktionistisch kritisiert, und im schlimmsten Fall setzten sie sich den Vorwurf aus, dass sie dem bestehenden System feindlich gegenüberstehen und Revolten anzetteln wollten. Und schreibt dann:

And it is indeed in such a spirit, let us confess, that we insist on class analysis, as that instrument required to recognize the inhuman, abstract and unearthly reductions forced onto people and planet by an economic system founded on a constitutive state of civil war, even if, today, this is a class war waged effectively only from above. ${ }^{23}$

Seit der Herausbildung des ssocial web gibt es eine Alternative zu staatlichen Medien oder zu privatwirtschaftlichen Medienkonzernen. Seit der Herausbildung des ssocial web ist klar geworden, dass dieses Medium nicht auf Vermittler und sgate keeper angewiesen ist, dass es einen gegenseitigen und unhierarchischen Austausch zwischen speers ermöglicht. Ein solches Medium hat im globalen Kontext bisher nicht existiert. Inzwischen sind Auseinandersetzungen über die Nutzung des Internets in vollem Gang. Dies betrifft erstens Fragen der Überwachung und Kontrolle der Privatsphäre von Internet NutzerInnen, sowohl seitens des Staats wie auch seitens globaler

22 Alain Badiou, The Communist Hypothesis, London: Verso 2010, S. 5 . 
Medien- und Technologieunternehmen. Die Dringlichkeit dieser Fragen muss seit den Enthüllungen von Edward Snowden nicht weiter erläutert werden. Dies betrifft zweitens Fragen der Kommodifizierung des Internets. Wie können wir das Internet als Gemeingut schützen und unterstützen? Drittens geht es um Fragen der Regulierung des Internets. Schließlich geht es viertens um Fragen der Legalität und Legitimität von neuen Formen von Netzpolitik und digitalem Aktivismus. Dies zeigen etwa Auseinandersetzungen über die Methoden der Hackergruppe Anonymous. Wer darf sich wie in der Auseinandersetzung um Informationshoheit beteiligen?

Dies sind Debatten nicht nur über die Zukunft des Internets, sondern über die Zukunft unserer Gesellschaft. Denn das Internet, dies kann nicht genug betont werden, ist nicht nur eine Technologie oder ein Medium, sondern vor allem ein sozialer Raum. Als solcher ist es auch ein soziales Experimentierfeld. Initiativen, Institutionen und Projekte, die im digitalen Raum Alternativen eruieren, gibt es zuhauf auf globaler, nationaler, regionaler, und lokaler Ebene. Diese Experimente finden in allen gesellschaftlich relevanten Feldern statt. Viele dieser Initiativen sind eine Form von rakademischem Aktivismus ${ }^{24}$ Was sich herausbildet, ist eine Form von marxistischer Medientheorie, die nicht nur Kritik an den bestehenden Verhältnissen sucht, sondern die sich aktiv an der Suche nach postkapitalistischen Lösungen beteiligt. Oder mit Marx: die nicht interpretieren, sondern verändern will. Es ist kein Zufall, dass im digitalen Kapitalismus dieser rakademische Aktivismus besonders auffällig in der Medien- und Kommunikationsforschung zu verorten ist. Es ist die logische Folge einer Entwicklung, in der Medien, Kommunikation und Information ins innerste Zentrum unserer kapitalistischen Gesellschaft gerückt sind.

24 Andreas Wittel, Digital Transitions, Saarbrücken: Lambert 2016, S. 73-85. 